\title{
A FUNÇÃO DE "COBB-DOUGLAS", A PARTIR DO MODELO MATEMÁTICO COM ERRO ADITIVO
}

\section{Manuel Luiz Figueirôa \\ Economista}

\author{
Orientador: Prot. Dr. F. Pimentel Gomes
}

Dissertaçào apresentada à Escola Superior de Agricultura "Luiz de Quelroz", da Universidade de Sảo Paulo, para obtençào do Titulo de Mestre em Estatistica e Experimentaçào Agronòmica.

PIRACICABA

Estado de São Paulo - Brasil

Dezembro, 1980 
11.

\section{A minha muther, \\ meus filhos $e$ \\ minha mãe,}

OFERECO.

\footnotetext{
A memória de meu pai

e meus irmãos,
}

DEDICO. 


\section{AGRADECIMENTOS}

Ao Dr. F. Pimentel Gomes, Chefe do Departamento de Matemática e Estatística da ESALQ, pela orientação na execução do presente trabalho e, principalmente, pela amizade e respeito.

A UFS - Universidade Federal de Sergipe, por sua polí tica de treinamento de docentes.

A AFA - Academia da Força Aérea, na pessoa do Cel.Av. Silvio da Gama Barreto Viana, Chefe do D.E., pela confiança e apoio. Aos Drs. Humberto de Campos e Roberto Simionato Moraes, do Departamento de Matemätica e Estatística da ESALQ, pelo apoio.

Ao Dr. Rodolfo Hoffmann, do Departamento de Economia e Sociologia Rural da ESALQ, pelas sugestões.

A Dr. Marli de Bem Gomes, do Departamento de Matemäti ca e Estatística da ESALQ, pela leitura dos originais, sugestōes e apoio.

Ao Prof. Sidney Jorge Schinaider, da AFA, pela colabo ração na execução dos cálculos experimentais.

A Coordenadoria de Aperfeiçoamento do Pessoal de Nível Superior (CAPES), através do PICD, pelo fornecimento da bolsa de estudos. 
A Srta. Maria Izalina Ferreira Alves, Secretária do De partamento de Matemática e Estatistica da ESALQ, pela datilografia do trabalho.

A todos que, direta ou indiretamente, colaboraram para a realização deste trabalho. 
Pág.

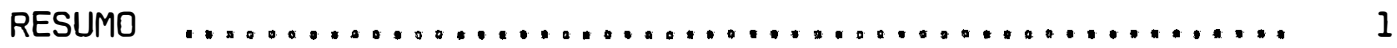

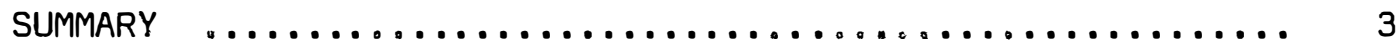

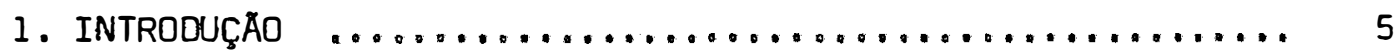

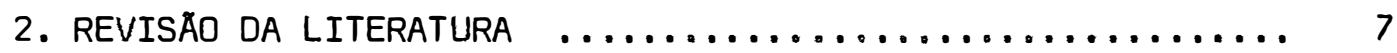

3. MATERIAL E METODOS $\ldots \ldots \ldots \ldots \ldots \ldots \ldots \ldots \ldots \ldots \ldots \ldots \ldots$

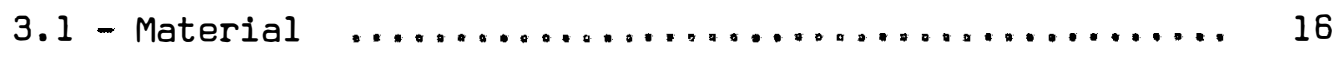

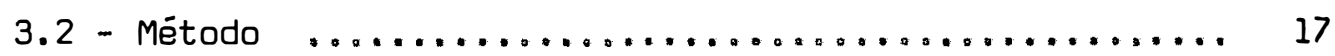

3.2 .1 - Função de Cobb-Douglas .............. 17

3.2 .1 .1 - Modelo I $\ldots \ldots \ldots \ldots \ldots \ldots \ldots$

3.2 .1 .2 - Modelo II $\ldots \ldots \ldots \ldots \ldots \ldots \ldots \ldots 18$

3.2.2 - Métodos para o ajustamento de funçöes de regressão transcendentes pelos mínimos quadra-

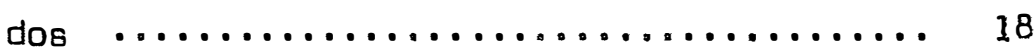

3.2.2.1 - Descrição do processo iterativo .. 19

3.2.2.2 - Aplicação do método de Newton e do método modificado de Gauss-Newton aos modelos em estudo ........ 20

3.2.2.2.1 - Caso de uma só variāvel independente .... 20

3.2.2.2.2 - Caso de duas variáveis independentes ..... 25

3.2.2.2.3 - Generalização do processo $\ldots \ldots \ldots \ldots \ldots 30$ 
vi.

Päg.

3.2.2.2.4 - Estimativa da dose eco nómica $\ldots \ldots \ldots \ldots \ldots .34$

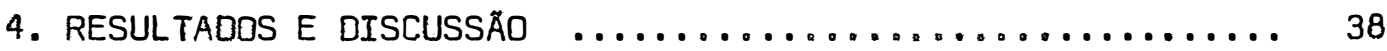

4.1 - Modelos com Erro Multiplicativo ............. 38

4.2 - Modelo I - Com Erro Aditivo ................ 40

4.3 - Modelo II - Com Erro Aditivo ................ 44

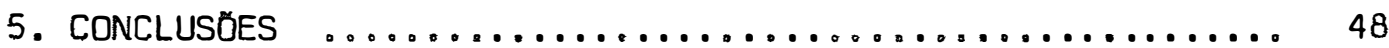

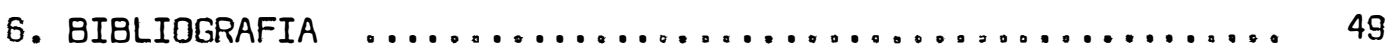

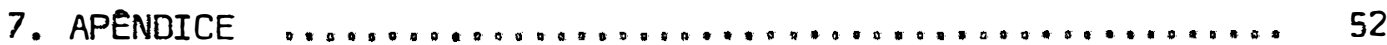


1.

\section{RESUMO}

Este trabalho apresenta um processo mais convincente do que o usual, para a estimativa dos parâmetros da função de Cobb-Doug las.

Alcançamos este objetivo adotando procedimento itera tivo através do método de Newton e do método modificado de Gauss-Newton, aplicados ao modelo

$$
Y_{i}=A X_{i}^{B}+e_{i}
$$

e concluimos, pelo critério de menor soma de quadrados dos desvios da regressão, a superioridade do procedimento que sugerimos. A resposta favorável foi obtida pelos dois métodos, o de Newton e o modi ficado de Gauss-Newton.

A seguir, repetimos o estudo para o modelo

$$
Y_{1}=A X_{11}^{B_{1}} X_{12}^{B_{2}}+e_{1}
$$


e concluimos, pelo mesmo critério, a superioridade do procedimento sugerido. Desta feita a resposta favorável foi observada apenas para o método modificado de Gauss-Newton, pois, quando da aplicação do método de Newton, não houve convergência nas estimativas dos parâmetros, com os valores iniciais usados.

Apresentamos, ainda, förmulas que permitem a generalização do processo, isto é, a repetição do estudo para o modelo

$$
y_{i}=A x_{11}^{B_{1}} x_{i 2}^{B_{2}} \ldots x_{i n}^{B_{n}}+\varepsilon_{i}
$$

ou

$$
Y_{i}=A \prod_{h=1}^{n} x_{i h}^{B_{h}}+e_{i},
$$

$\operatorname{com} 1=1,2, \ldots, N$.

No estudo da dose econômica de nutrientes apresentamos a fórmula correspondente ao primeiro modelo. Para o segundo e para o modelo generalizado, indicamos os sistemas cujas soluções apresentam as doses económicas aconsel háveis de nutrientes, se satis feita a condição de ser definida negativa a diferencial segunda da receita líquida. 


\section{SUMMARY}

This paper discusses the estimation, by the method of least squares, of parameters of the Cobb-Douglas function with additive error, instead of the usual multiplicative error with use of logarithms. The estimates are obtained both by Newton's and Gauss-Newton's method. These methods were applied in detail for models

$$
Y_{i}=A X_{i}^{B}+e_{i}
$$

and

$$
Y_{1}=A x_{11}^{B_{1}} X_{12}^{B_{2}}+e_{1}
$$

with complete discussion of an example for each model.

The main conclusions reached in this research were the following: 
1 - For the first model, with one independent variable, the additive error led to a better adjustment than the tradicional multiplicative error, with good results, either by Newton's or by Gauss-Newton's metiod.

2 - For the second model, with two independent variables, convergence was obtained only for the Gauss-Newton's method, and led also to a better adjustment than the tradicional procedure.

3 - Convergence, when present, was rather quick, so that only a few iterations were necessary. 
5.

\section{INTRODUÇÃO}

Como ė do conhecimento dos estudiosos do assunto, a função de Cobb-Douglas tem sido ajustada a dados de observação atra Vés de modelo matemätico com erro multiplicativo.

o procedimento geral tem sido o de adotar o modelo

$$
y=\alpha x_{1}^{\beta_{1}} x_{2}^{\beta_{2}} x_{3}^{\beta_{3}} \ldots x_{n}^{\beta_{n}}(1+\varepsilon),
$$

$\operatorname{com} \alpha>0, x_{h}>0(h=1,2, \ldots, n)$

Após aplicação de logarítmo, obtemos

$$
\begin{aligned}
\log Y=\log \alpha & +\beta_{1} \log X_{1}+\beta_{2} \log X_{2}+\ldots+\beta_{n} \log X_{n}+ \\
& +\log (1+\varepsilon) .
\end{aligned}
$$

onde, para

$$
\begin{aligned}
& y=\log Y \\
& a=\log \alpha
\end{aligned}
$$




$$
\begin{aligned}
x_{h} & =\log x_{h} \\
e & =\log (1+\varepsilon),
\end{aligned}
$$

temos

$$
y=a+\beta_{1} x_{1}+\beta_{2} x_{2}+\ldots+\beta_{n} x_{n}+e,
$$

que è uma equação de regressão linear múltipla, de aplicação bem có nhecida.

Este método tem apresentado resultados que, apesar de aceitos como satisfatórios, guardam uma certa desconfiança no seu uso.

A restrição que se faz, decorre do fato de o erro adotado ser multiplicativo, o qual, após transformação logarítmica, se torna aditivo.

0 que se pretende com este trabalho é adotar no mode lo matomático o erro aditivo, fazer a análise da variancla e estimar a dose econômica aconselhảvel.

Inicialmente, estudamos o modelo na sua forma mais simples,

$$
Y_{1}=A X_{1}^{B}+e_{1}
$$

para, logo em seguida, repetirmos o estudo com o modelo

$$
Y_{1}=A X_{11}^{B_{1}} X_{i 2}^{B_{2}}+e_{i}
$$




\section{REVISÃO DA LITERATURA}

0 interesse pelas funções de produção vem desde a época dos clássicos, com Adam Smith, David Ricardo e Malthus (GIRÃo, 1965\}. De acordo com esse autor, quem primeiro sugeriu uma forma al gébrica para a função de produção agrícola foi Knut Wicksell, em 1916, com a função proposta

$$
p=a^{\alpha} b^{\beta} c^{\gamma}
$$

onde $\alpha+\beta+\gamma=1$, $\underline{p}$ designa a produção anual e $\underline{a}$, 므 $\underline{c}$ são as quan tidades utilizadas, respectivamente, dos fatores de produção terra, trabalho e capital.

Entretanto, em dados da indústria manufatureira americana no período de 1899 a 1922, Charles Cobb e Paul Douglas, em 1928, propōs a função

$$
P^{\prime}=b L^{k} C^{1-k}
$$


onde $P^{\prime}$ era o Indice previsto para a produção durante o período em estudo; L o Indice do número médio anual de assalariados; e C o indice dos valores do capital fixo na indústria, previamente deflacio nados. Salientemos, ainda, que já em 1909 MITSCHERLICH (1909) apresentava a sua famosa lei, que liga a produção $Y$ a uma dose $X$ de nutriente adicionado ao solo.

HARTLEY (1961) desenvolveu um novo processo, o método modificado de Gauss-Newton, para estimação de parâmetros em equa ções transcendentes. Partindo de estimativas preliminares para cada um dos parâmetros, o autor obteve estimativas sucessivas para todos eles, de tal forma, que os acréscimos a estimativas de ordens anteriores são comparados com um grau de precisão pré-estabelecido. In cia-se, assim, um processo iterativo, até se obterem estimativas eficientes para todos os parâmetros que compõem o modelo.

GIRÃO (1965) realizou um trabalho sobre a função de produção de Cobb-Douglas e a análise inter-regional da produção agrícola, através da comparação das produtividades dos fatores de produção, em grupos regionais de explorações agrícolas. Apresentou as vantagens e as desvantagens do tipo de função utilizada, os aspectos metodológicos próprios da regressão linear múltipla, a interpretação estatística de regressões obtidas a partir dos dados re ferentes às explorações agrícolas em cinco regiões diferentes de Por tugal e os fundamentos teóricos em que baseou a análise inter-regio nal efetuada. 
PIMENTEL GOMES e ZAGATTO (1967) estudaram os aspectos econômicos da adubação, apresentaram a conceituação dos agrega dos econômicos da produção, e aplicaram a função correspondente com uma, duas, três e $\underline{n}$ variáveis independentes, respectivamente. Indicaram métodos de obtenção dos mapas de isoquantas e isóclinas. Estu daram também a quantidade ótima a ser aplicada do fator produtivo sob várias hipóteses, como sejam: capital ilimitado, capital limita do, etc. Finalmente chamaram a atenção para um conjunto de dificuldades que podem ocorrer no estudo económico por polinômios de ensaios de adubação.

ENGLER (1968) estabeleceu uma função de produção, es pecificando as relações estruturais entre o valor da produção total e os fatores empregados no processo produtivo. Adotou a função de Cobb-Douglas com erro multiplicativo e determinou:

a) os coeficientes de elastı́cidades de produção;

b) os produtos marginais dos fatores de produção terra, trabalho e capital;

c) e as taxas marginais de substituição entre esses fatores.

Concluiu que a produção agrícola, nos dois municípios estudados (Guarel e Itapetininga), apresentou rendimentos de escala.

MALINVAUD (1969) explicou que a função de produção de uma empresa representa as dificuldades tecnológicas que se impuseram a essa unidade de produção. Ela determina as quantidades de 
produtos que podem ser obtidos a partir de qualquer combinação de fatores colocados no processo produtivo. Afirmou que a função de Cobb-Douglas parece adequada, como primeira aproximação, em numerosos casos de relação insumo-produto. Disse ainda que a estimativa de funções de produção, a partir de dados relativos às empresas de um mesmo tipo, requer a tomada do conjunto das relações que expl1cam os Insumos e produtos e é, então, um modelo de equações múlt1plas que intervirão ma1s frequentemente e a forma desse modelo varla de uma situação para outra.

SIMONSEN (1971) definiu função de produção como indicativa da produção máxima que se pode obter através de um processo de produção e, este, através do concelto matemático de funcional. Apresentou uma função de Cobb-Douglas homogênea e afirmou que, no ca so de fatores substituíveis, é a que melhor se aplica na agricultura. No entanto, há, sem dúvida, situações em que a função de Cobb-Douglas não pode, pelas suas propriedades matemáticas, representar adequadamente o fenómeno.

PINHEIRO (1972) ut1lizou o modelo econométrico de Cobb-Douglas na análise da produção da pecuáría bovina do município de Botucatu, Estado de São Paulo. Selecionou do1s, dentre doze mode los alternativos, que melhor representaram as relações de produção da pecuáría bovina na região estudada. Usou também o modelo de erro multiplicativo, como fizeram, aliás, todos os demais trabalhos menclonados a seguir que aplicaram a mesma função. 
NEVES (1972) utilizou o modelo Cobb-Douglas, para es timar função de produção que represente as relações insumo-produto, para a exploração leiteira na região da Média Noroeste,composta dos municípios de Lins, Cafelândia, Promissão, Guaiçara, Sabino e Getulina. Analisou comparativamente a produção obtida nos períodos de estação seca ou chuvosa.

SILVA (1973) utilizou o modelo econométrico de Ulveling-Fletcher,procurando conhecer os efeitos do crédito rural na par tilha e produtividade dos recursos produtivos nas propriedades agri colas da Divisão Regional Agrícola de Ribeirão Preto, Estado de São Paulo. Desse estudo obteve algumas conclusões como:

a) os pequenos agricultores dificilmente têm acesso ao crédito rural;

b) os agricultores especializados em culturas anuais estão acen tuadamente voltados para o mercado;

c) o valor da terra, representando $64,24 \%$ do capital agrário, é o seu principal componente.

CRÓCOMO (1974) ajustou uma função de produção do t1po Cobb-Douglas a culturas de milho e de soja, através de dados obtidos de 120 entrevistas diretas com os agricultores dos municipios de Guaira, Jardinópolis e Sales de Oliveira, no Estado de São Paulo, no ano agrícola 1971/1972. Na estimativa dos parâmetros da função de produção, utilizou o erro multiplicativo e a transformação logarítmica. 
CAMARGO (1974) estudou a eficiência do uso dos recur sos nas culturas de soja e algodão utilizando na estimativa de funções de produção o modelo de Cobb-Douglas e o de Ulveling-Fletcher, e comparau os resultados obtidos. Concluiu que a função de Ulveling-Fletcher ajustou-se melhor aos dados da cultura de soja. Embora no caso do algodão o coeficlente de determinação fosse praticamente da mesma ordem para as duas funções, a de Ulveling-Fletcher se revelou mais flexivel, oferecendo maiores possibilidades para análise. No entanto é duvidoso que se possa julgar a conveniència de uma equação de regressão apenas pelo coeficiente de determinação, pois as propriedades matemáticas são muito mais importantes.

SILVEIRA (1976) utilizou o método desenvolvido por HARTLEY (1961) no estudo do comportamento dos modelos de crescimento de Von Bertalanffy, Brody, Gompertz e Logistico, quando aplicados a dados de crescimento de bovinos da raça Ibagé.

NOJIMOTO (1976) estudou e analisou todos os trabalhos sobre função de produção até essa data. Suas principais conclü söes foram:

a) os erros que se cometem nos levantamentos de dados são tantos que comprometem os resultados das pesquisas;

b) os trabalhos empíricos utilizam förmulas inadequadas para estimar o produto marginal e isto leva a conclusões falsas ou irrelevantes; 
c) os intervalos de confiança dos parâmetros e dos produtos mar ginais são tão amplos que as estimativas têm pouco valor como instrumento de previsão.

MELO (1976) estudou a equação de Mitscherlich e também a forma adotada por Baule, esta com duas ou três variáveis inde pendentes, em 50 ensaios fatoriais $3^{3}$ de adubação $\operatorname{com} N, P$ e $K$ em milho, instalados em terra roxa de Ribeirão Preto, SP. Utilizou o método modificado de Gauss-Newton para ajustar funções de regressão transcendentes pelos mínimos quadrados, tendo concluido que houve, para os casos estudados, efeito altamente significativo para a regressão e que o mesmo não ocorreu para os desvios de regressão.

HOFFMANN e VIEIRA, em livro publicado em 1977, incluiram interessante capítulo sobre a regressäo assintótica, onde estudaram a estimação, pelos métodos de Newton e de Gauss-Newton, de parâmetros da equação $Y_{i}=\alpha+\beta \rho^{X_{i}}+e_{i}$, uma das formas da lei de Mitscherlich.

PERRE DA SILVA (1979) usou o método modificado de Gauss-Newton para estimar os parâmetros da equação de Mitscherlich na sua segunda aproximação e calculou as doses economicamente aconselháveis de nutrientes. Para esse fim usou:

a) um ensaio, fictício com 13 nIveis de nutriente e 4 repetições, em delineamento inteiramente casualizado;

b) Um ensaio de adubação de trigo, instalado em Londrina, PR, por pesquisadores do IAPAR e da EMBRAPA, no delineamento de 
blocos ao acaso com parcelas subdivididas.

ENGLER (1978), adotando o modelo econométrico de Cobb-Douglas com erro multiplicativo:

a) estabeleceu uma função de produção agregada, tendo como uni dade de análise as 48 sub-divisões regionais do Estado de São Paulo, e tendo por base de informação os dados obtidos em entrevistas diretas com 6.996 agricultores constantes de amostra em corte seccional do Instituto de Economia Agricola e da Coordenadoria de Assistência Técnica Integral(CATI) da Secretaria de Agricultura do Estado de São Paulo;

b) analisou as diferenças regionais na produção e na produtiví dade agrícola e suas implicações para o processo de desenvolvimento econômico;

c) analisou a eficiēncia econômica da agricultura na distribui ção dos recursos produtivos;

d) estimou a contribuição de determinantes do capital humano, como educação formal, assistēncia técnica, extensão e experiència do agricultor, para a produção agrícola do Estado. COSTA LIMA (1980), na procura de uma fórmula de adubação economicamente aconselhável para a cultura da mandioca, no Es tado do Ceará, utilizou 22 ensaios fatoriais $3^{3}$ incompletos de adubação com N. P e K, do projeto EMBRATER/FAO/MA, instalados no perio do de 1972/76. Aos dados experimentais aplicaram-se os modelos de regressão polinomial e de regressão assintótica. Para o modelo as- 
15.

sintötico usou-se o método modificado de Gauss-Newton, desenvolvido POI HARTLEY (1961). 
16.

\section{MATERIAL E METODOS}

\section{1 - Material}

Os dados usados para o estudo do modelo

$$
Y_{1}=A \cdot X_{1}^{B}+e_{1}
$$

foram os mesmos utilizados por PERRE DA SILVA (1978, p. 93), excluUI do o nivel zero em virtude de ocorrer impedimento matemático de aplicá-1o no desenvolvimento teórico no caso da função de Cobb-Douglas. Segundo a autora as repetições foram geradas através do modelo matemático $Y_{i}=m+t_{1}+e_{i}$, onde tomou $m=100$ como média geral do experimento e variância $\sigma^{2}=V\left(e_{i}\right)=12$.

A variável $x_{1}$ representa os níveis de tratamentos, que foram 0,$2 ; 0,4 ; 0,6 ; 0,8 ; 1,0 ; 1,2 ; 1,4 ; 1,6 ; 1,3 ; 2,0 ; 2,2$ e 2,4 (depois de excluldo o zero). 
Para o modelo

$$
Y_{i}=A X_{i 1}^{B_{1}} X_{i 2}^{B_{2}}+e_{i}
$$

o autor gerou dados que foram obtidos da função

$$
y_{i}=68,2857 x_{i 1}^{0,2168} x_{i 2}^{0,3542}+e_{i}
$$

e se encontram na Tabela 2 do Apêndice, onde o erro tem distribuição normal de média zero e variância quatro. Os valores de $e_{i}$ foram extraídos da tabela de nümeros aleatórios de DIXON e MASSEY [1951. pp. 355 a 359). As variáveis $x_{i 1}$ e $x_{i 2}$ representam os níveis de tra tamentos que foram 0,$1 ; 0,3 ; 0,5 ; 0,7 ; 0,9 ; 1,1 ; 1,3 ; 1,5 ; 1,7 ; 1,9 ;$ 2,$1 ; 2,3$ e 0,$2 ; 0,4 ; 0,6 ; 0,8 ; 1,0 ; 1,2 ; 1,4 ; 1,6 ; 1,8 ; 2,0 ; 2,2 ;$ 2,4, respectivamente.

\section{2 - Mētodo}

\subsection{1 - Função de Cobb-Douglas}

Os modelos em estudo serão utilizados para determinar a produção através da função de Cobb-Douglas.

A variável $Y_{i}$ representa a produção obtida com as dosagens $x_{i}$ de um nutriente.

\subsubsection{1 - Modelo}

$$
y_{i}=A X_{i}^{B}+e_{i}
$$


onde: $A$ e $B$ são parâmetros;

$e_{i}$ é o erro aleatórı, que se supöe $e_{i} \cap N\left(0, \sigma^{2}\right)$.

$$
\begin{aligned}
& 3.2 .1 .2 \text { - Modelo } 11 \\
& \qquad Y_{i}=A X_{i 1}^{B_{1}} X_{i 2}^{B_{2}}+e_{i},
\end{aligned}
$$

onde $A_{2} B_{1}$ e $B_{2}$ são paràmetros;

$e_{i} \cap N\left(0, \sigma^{2}\right)$

3.2.2. - Mẽtodos para o ajustamento de funções de regressão transcendentes pelos minimos quadrados

Os parámetros, para cada um dos do1s modelos anterio res, serão estimados através do método de Newton e do método modif 1 cado de Gauss-Newton (HOFFMANN e VIEIRA, 1977).

Frequentemente, nos deparamos com o problema de deter minar a relação entre $Y_{1}$ e os valores $X_{1 h}, X_{2 h}, \ldots, X_{k h}(h=1,2, \ldots$ $\ldots, n)$

Na malorla das vezes, admite-se uma relaçäo funcional conhecida, escrita sob a forma de uma função de regressão,

$$
f(x, \theta)=f\left(x_{1}, x_{2}, \ldots, x_{n} ; \theta_{1}, \theta_{2}, \ldots, \theta_{m}\right),
$$

onde os parámetros $\theta_{1}, \theta_{2}, \ldots, \theta_{m}$ devem ser estimados. Nestas condições, è necessárı determinar um vetor $\theta$, para o qual a soma de quadrados 


$$
Q(\theta)=\sum_{i=1}^{N}\left[Y_{i}-f\left(x_{i}, \theta\right)\right]^{2}
$$

seja minima. AI, $x_{i}$ representa o vetor $\left(x_{i 1}, x_{i 2}, \ldots, x_{i n}\right)$ e $\theta$,por sua vez, é o vetor $\left(\theta_{1}, \theta_{2}, \ldots, \theta_{m}\right)$.

\subsubsection{1 - Descrição do processo iterativo}

Consideremos que ${ }_{0} \hat{\theta}_{1},{ }_{0} \hat{\theta}_{2}, \ldots, 0_{0} \hat{m}_{\text {sejam }}$ estimativas preliminares dos parāmetros $\theta_{k}(k=1,2, \ldots, m)$ (posteriormente ve remos como calculá-las).

O primeiro passo, é obter correções para os elementos ${ }_{0} \bar{\theta}_{k}$ do vetor inicial.

Dada uma amostra de $\underline{N}$ valores de $X_{1}$ e $Y_{i}$ e estabelecidos os valores das estimativas preliminares ${ }_{0} \bar{\theta}_{k}$ obtemos as correções $\Delta \bar{\theta}_{k}$. Se essas correções não forem despreziveis, obtemos ${ }_{1} \hat{\theta}_{k}=$ $={ }_{0} \hat{\theta}_{k}+\Delta \hat{\theta}_{k} \cdot A$ seguir, utilizando ${ }_{1} \hat{\theta}_{k}$ como novas estimativas preliminares, os cálculos são refeitos, obtendo-se novas corrȩ̣ões.

Admitindo-se que o processo seja convergente, isto é, que os valores absolutos das correções tendam para zero, os cälculos são iterados até que as correções $\Delta \hat{\theta}_{k}$ sejam consideradas despre zÍveis. Chega-se, assim, às estimativas de quadrados mínimos, que indicamos por $\hat{\theta}_{1}, \hat{\theta}_{2}, \ldots, \hat{\theta}_{m}$. 
3.2.2.2 - Aplicação do método de Newton e do método modificado de Gauss-Newton aos modelos em estudo

O método modificado de Gauss-Newton serā apresentado como um caso particular do método de Newton, isto é, nas equações de Newton admitiremos algumas parcelas como desprezíveis, e, obteremos desta forma as equaçōes do método modificado de Gauss-Newton.

\subsubsection{1 - Caso de uma só variàvel inde pendente}

Seja

$$
Y_{1}=A X_{i}^{B}+e_{i},
$$

$\operatorname{com} A>0, B>0, X_{1}>0$, para $1=1,2, \ldots N$.

Sejam a e $\underline{b}$ as estimativas de $A$ e $B$, respectivamente, obtidas com base em uma amostra de tamanho $\underline{N}$ de $X_{i}$ e $Y_{i}$, de acordo com o método dos quadrados mínimos.

Temos,

$$
\begin{aligned}
& Y_{1}=A X_{1}^{B}+e_{1}, \quad A>0 ; B>0 ; X_{i}>0 ; \\
&(i=1,2, \ldots, N)
\end{aligned}
$$

e,

$$
z=\Sigma e_{i}^{2}=\sum_{i=1}^{N}\left(Y_{i}-A X_{i}^{B}\right)^{2}
$$

Diferenciando (1) obtemos:

$$
d z=\frac{\partial z}{\partial A} d A+\frac{\partial z}{\partial B} d B,
$$


onde,

$$
\begin{aligned}
& \frac{I}{2} \frac{\partial z}{\partial A}=-\sum\left(Y_{1}-A X_{1}^{\theta}\right) x_{1}^{B} \\
& \frac{I}{2} \frac{\partial z}{\partial B}=-A \sum\left(Y_{1}-A X_{1}^{B}\right) X_{1}^{B}\left(L X_{i}\right)
\end{aligned}
$$

Ai indicamos por $L X_{1}$ o logarítmo neperiano de $X_{1}$. Portanto, as equações normais são:

$$
\left\{\begin{array}{l}
\Sigma\left(Y_{1}-Q_{1}\right) x_{1}^{D}=0 \\
\Sigma\left(Y_{1}-P_{1}\right) X_{i}^{D}\left(L X_{1}\right)=0
\end{array}\right.
$$

$\operatorname{com} P_{1}=a X_{i}^{b}$

0 fato de em (4) e (5) usarmos a e b destaca o uso das estimativas dos parâmetros nas equações normais.

E evidente que estamos admitindo a condição suficien te para a e $\underline{b}$ serem pontos de mínimo, isto $\dot{e}$, que a diferencial segunda de (1) para a e $\underline{b}$ seja definida positiva.

Admitindo-se existentes as condições para o desenvol vimento de uma função pela fórmula de Taylor, e aplicando-se esse procedimento às equaçöes (4) e (5), temos o sistema

h

$$
\left[\Sigma x_{i}^{2 b_{0}}\right] \Delta a+\left[\begin{array}{c}
a_{0} \Sigma x_{i}^{2 b_{0}}\left(L X_{i}\right)- \\
-\Sigma\left(Y_{i}-Y_{i}^{*}\right) x_{i}^{b_{0}}\left(L X_{i}\right)
\end{array}\right] \Delta b=\Sigma\left(Y_{i}-Y_{i}^{*}\right) x_{i}^{b_{0}}
$$




$$
\left[\begin{array}{c}
\Sigma x_{1}^{2 b_{0}}\left(L X_{1}\right)
\end{array}\right] \Delta a+\left[\begin{array}{c}
a_{0} \Sigma x^{2 b_{0}}\left(L X_{1}\right)^{2}- \\
-\Sigma\left(Y_{1}-Y_{i}^{*}\right) x_{1}^{b_{0}}\left(L X_{1}\right)^{2}
\end{array}\right] \Delta b=\Sigma\left(Y_{1}-Y_{i}^{*}\right) x_{1}^{b_{0}}\left(L X_{i}\right)
$$

onde, $a_{0} e b_{0}$ são estimativas preliminares, $e r_{i}^{*}=a_{0} x_{1}^{b_{0}}$.

$$
\text { Se fizermos } f_{1}=x_{1}^{2 b_{0}}, g_{1}=\left(y_{1}-y_{i}^{*}\right) x_{1}^{b_{0}} \text { e } \ell_{1}=L X_{1} \text {, }
$$

esse sistema, escrito na forma matricial, é:

$$
\left[\begin{array}{ccc}
\Sigma f_{1} & a_{0} \sum f_{1} \ell_{1}-\Sigma g_{1} \ell_{1} \\
\Sigma f_{1} l_{1} & a_{0} \sum f_{1} \ell_{1}^{2}-\Sigma g_{1} l_{1}^{2}
\end{array}\right]\left[\begin{array}{l}
\Delta a \\
\Delta b
\end{array}\right]=\left[\begin{array}{c}
\Sigma g_{1} \\
\Sigma g_{1} l_{1}
\end{array}\right]
$$

Essas são as equaçōes correspondentes do método de

Newton.

Ocorre, porêm, que a primeira matriz acima pode ser decomposta da seguinte forma,

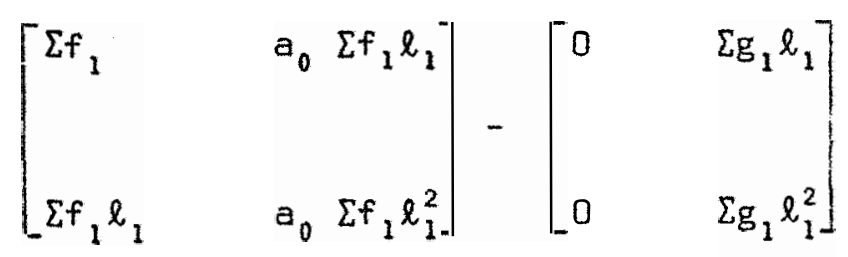

Os elementos da segunda matriz ou são nulos ou são somas ponderadas de desvios. Então, os valores absolutos dos elemen tos dessa matriz devem ser pequenos em comparação com os valores ab solutos dos elementos da primeira matriz. Assim sendo, temos, aproximadamente: 


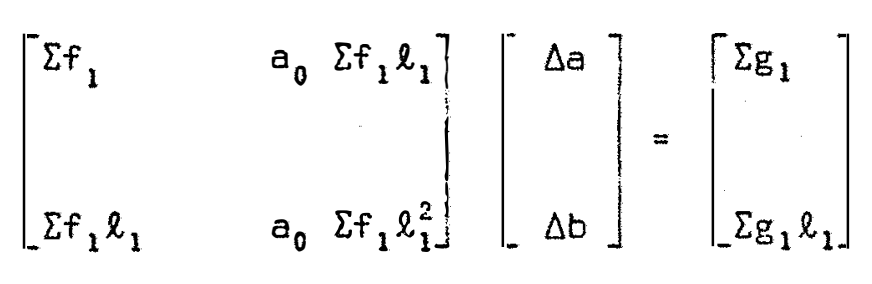

Tais são as equações do método modificado de Gauss-Newton.

Admite-se que a primeira matriz do primeiro membro de cada um dos sistemas $(\alpha)$ e ( $\beta$ ) seja não-singular, de onde resulta serem eles compatíveis e determinados, isto é, com uma só solução,

Dada uma amostra de tamanho $N$ de pares $\left(X_{1}, Y_{1}\right)$ e estabelecidos os valores das estimativas preliminares $a_{0}$ e $b_{0}$, obtemos com as soluções dos sistemas $\alpha$ e $\beta$, nas aplicações dos métodos de Newton ou do modificado de Gauss-Newton, os valores de $\Delta a$ e $\Delta$ t. Se essas correções não forem desprezíveis obtemos $a_{1}=a_{0}+\Delta a$, $b_{1}=b_{0}+\Delta b$. A seguir, utilizando $a_{1} e b_{1}$ como novas estimativas preliminares, os cálculos são refeitos, obtendo-se novas correções de $\Delta a$ e $\Delta b$.

Admitindo-se que o processo seja convergente, isto é, que os valores absolutos das correções tendam a zero, os cálculos são repetidos até que as correções $\Delta a$ e $\Delta b$ sejam consideradas desprezíveis. Chega-se, assim, às estimativas de quadrados mínimos de $A$ e $B$, que indicamos por $\underline{a}$ e $\underline{b}$. 
Cälculo de estimativas preliminares $a_{0} e b_{0}$

0 método usado na obtenção das estimativas iniciais foi o seguinte.

$$
\begin{aligned}
& \text { Consideremos, } \\
& \qquad Y_{i}=A X_{i}^{\theta}\left(I+\varepsilon_{i}\right), \\
& \therefore \quad L Y_{i}=L A+B\left(L X_{i}\right)+L\left(I+\varepsilon_{i}\right) .
\end{aligned}
$$

Fazendo

$$
\begin{aligned}
y_{i} & =L Y_{i} \\
a & =L A \\
x_{i} & =L X_{i} \\
e_{i} & =L\left(I+\varepsilon_{i}\right)
\end{aligned}
$$

temos

$$
y_{i}=a+B x_{i}+e_{i}
$$

que è uma equação de regressão linear, cuja aplicação é bem conheci da.

Desta forma, obtivemos as estimativas preliminares $a_{0}$ e $b_{0}$, dadas por à e Ê, através das relações

$$
\begin{aligned}
& a=\exp \hat{a}, \\
& b=\hat{B} .
\end{aligned}
$$




\subsubsection{2 - Caso de duas variäveis inde- pendentes}

Seja

$$
Y_{1}=A X_{11}^{B_{1}} X_{12}^{B_{2}}+e_{i} \text {. }
$$

$\operatorname{com} A>0, B_{1}>0, B_{2}>0,1=1,2, \ldots, N, x_{i j}>0$, para $j=1,2$.

Sejam $a_{2} b_{1}$ e $b_{2}$ as estimativas de $A, B_{1}$ e $B_{2}$ respec tivamente, obtidas com base em uma amostra de tamanho $\underline{N}$ dos vetores $\left(X_{11}, X_{12}, Y_{1}\right)$, de acordo com o método de quadrados mínimos.

Temos,

$$
Y_{1}=A x_{11}^{B_{1}} x_{12}^{B_{2}}+E_{1} \text {, }
$$

e

$$
z=\sum_{i=1}^{N} e_{i}^{2}=\sum_{i=1}^{N}\left(Y_{i}-A x_{i 1}^{B_{1}} x_{i 2}^{B_{2}}\right)^{2}
$$

Diferenciando (6) obtemos:

$$
d z=\frac{\partial z}{\partial A} d A+\frac{\partial z}{\partial B_{1}} d B_{1}+\frac{\partial z}{\partial B_{2}} d B_{2},
$$

onde,

$$
\begin{aligned}
& \frac{1}{2} \frac{\partial z}{\partial A}=-\Sigma\left(Y_{i}-A x_{i_{1}}^{B_{1}} X_{i_{2}}^{B_{2}}\right) x_{i_{1}}^{B_{1}} x_{i_{2}}^{B_{2}} \\
& \frac{1}{2} \frac{\partial z}{\partial B_{1}}=-\sum\left(Y_{1}-A x_{i_{1}}^{B_{1}} x_{i_{2}}^{B_{2}}\right) A x_{i_{1}}^{B_{1}} X_{i_{2}}^{B_{2}}\left(L X_{i_{1}}\right) \\
& \frac{1}{2} \frac{\partial z}{\partial B_{2}}=-\sum\left(Y_{1}-A x_{i_{1}}^{B_{1}} X_{i_{2}}^{B_{2}}\right) A X_{i_{1}}^{B_{1}} X_{i_{2}}^{B_{2}}\left(L X_{i_{2}}\right)
\end{aligned}
$$


portanto, as equeçōes normats sãc:

$$
\left\{\begin{array}{l}
\Sigma\left(Y_{1}-P_{1}\right) x_{13}^{b_{1}} x_{12}^{b_{2}}=0 \\
\Sigma\left(Y_{1}-q_{1}\right) x_{12}^{b_{1}} x_{12}^{b_{2}}\left(L x_{11}\right)=0 \\
\Sigma\left(Y_{1}-P_{1}\right) x_{11}^{b_{1}} x_{12}^{b_{2}}\left(L x_{12}\right)=0
\end{array}\right.
$$

$\operatorname{com} P_{1}=a x_{11}^{b_{1}} x_{12}^{b_{2}}$.

D fato de en (7), (8) \& (9) usarmos $a, b_{1}$ e $b_{2}$ destaca o uṣo das estimatıvas dos parâmetros nas equaçöes normsis.

E evidente que estamos ach Itindo a condição suficlente para $a_{1} b_{1}$ e $b_{2}$ seren coordenadas de um ponto de mfnimo, 1sto $\dot{a}$, que a efferencial segunda de (6) para $a, b_{1}$ a $b_{2}$ seja definida pos 1 tIva.

Adritindo-se existentes as condlções para o desenvolvimento de una funçäo pela fórmula de Taylor, e aplicando-se esse procedimento às equaçöes $(7),(8)$ e $(9)$, temos o sistema:

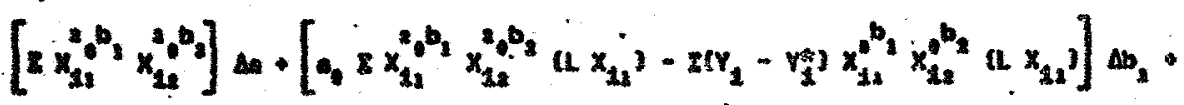

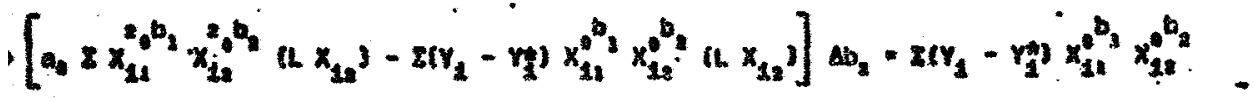

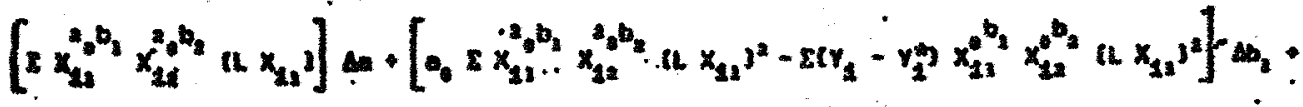

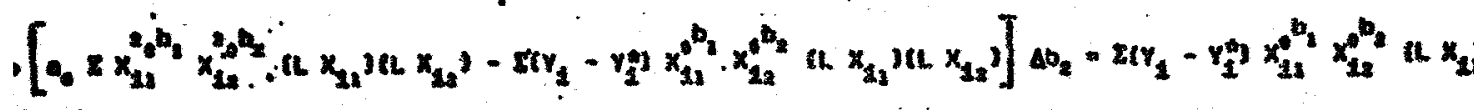




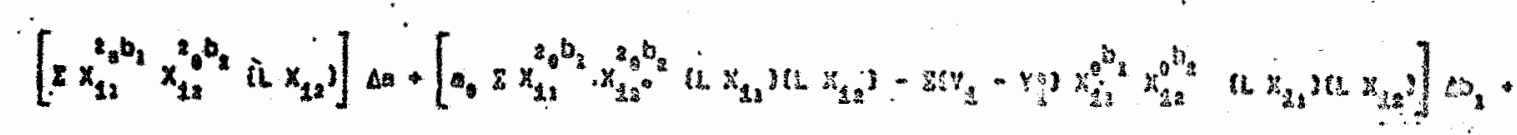

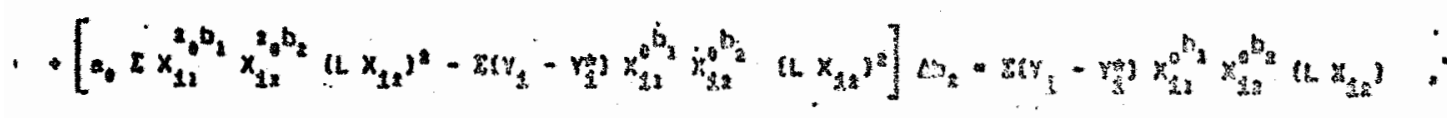

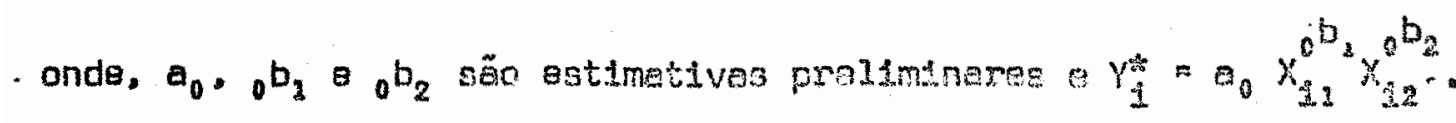

$$
\begin{aligned}
& f_{2}=x_{11}^{2} b_{12}^{b_{3}} x_{12}^{2_{0} b_{2}}, \quad g_{2}=\left(Y_{1}-Y_{1}^{*}\right) x_{11}^{0_{1}^{b_{1}}} x_{12}^{b^{b_{2}}}, \\
& l_{1}=L X_{11} \quad, \quad l_{2}=L K_{12} \quad,
\end{aligned}
$$

8sse sistemo, escrito ne forms metricial, se torna:

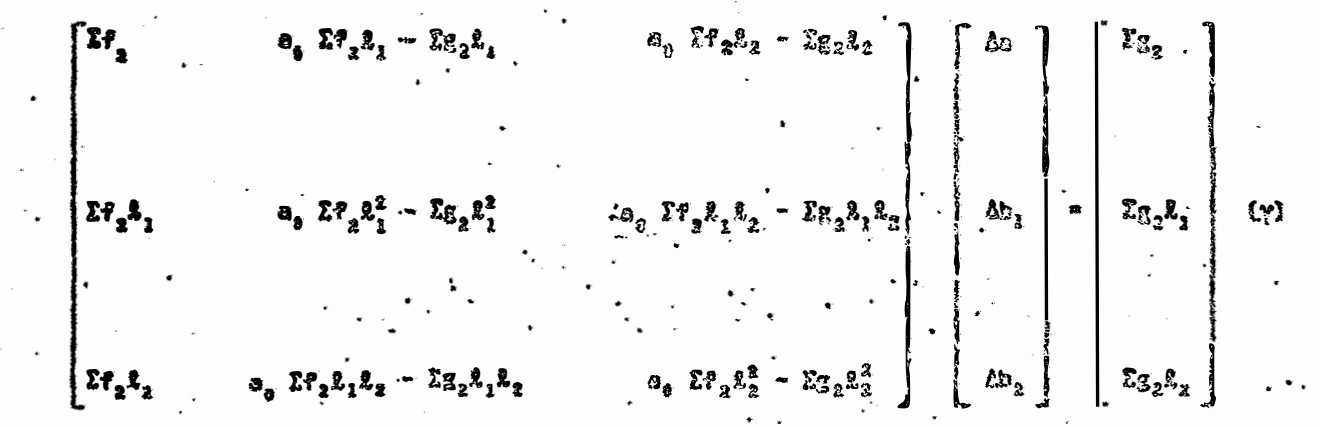

₹

$$
\text { Obtivemos, assim, as equ凶ęões correspondentes do mëto }
$$

do de Newton.

Dcorre qua, a primeira matriz acima, pode ser cbcomposta da seguinte forms: 


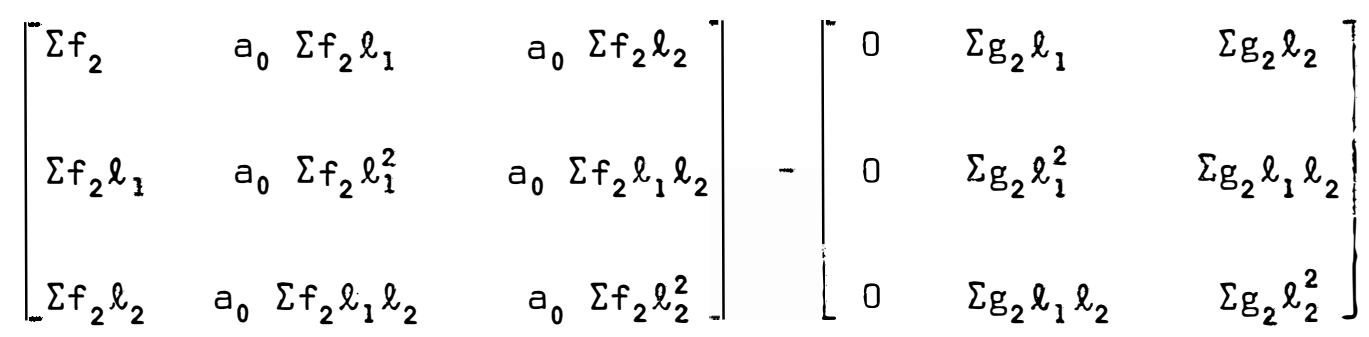

Os elementos da segunda matriz ou são nulos ou são so mas ponderadas de desvios. Então, os valores absolutos dos elementos dessa matriz devem ser pequenos em comparação com os valores ab. solutos dos elementos da primeira matriz. Assim sendo, temos, aproximadamente:

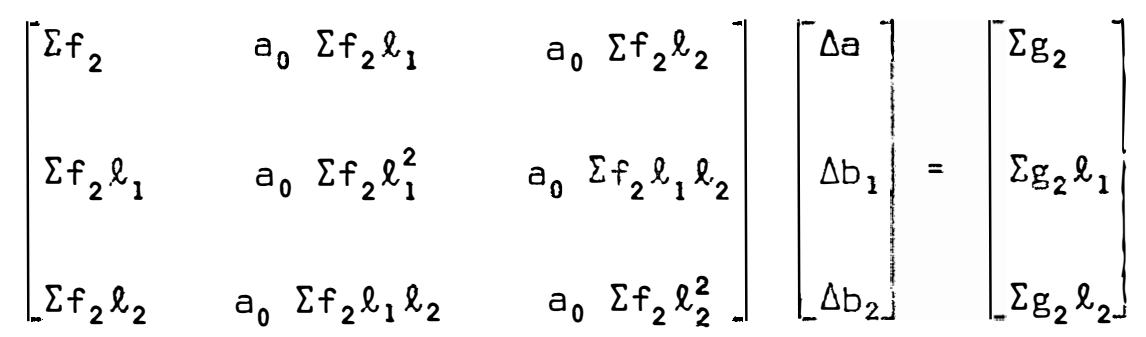

Estas são as equações correspondentes ao método modificado de Gauss-Newton.

Admite-se que a primeira matriz do primeiro membro de cada um dos sistemas $(\gamma)$ e $(\delta)$ seja não-singular, de onde resulta se rem eles compatíveis e determinados, isto é. com uma só solução.

Dada uma amostra de tamanho $\underline{N}$ de vetores $\left[X_{i 1}, X_{i 2}, Y_{i}\right]$ e estabelecidos os valores das estimativas preliminares ${ }_{0} a_{0} b_{1} e$ ${ }_{0} \mathrm{~b}_{2}$, obtemos, com as soluções dos sistemas $(\gamma)$ e $(\delta)$, nas aplica- 
ções dos métodos de Newton ou do modificado de Gauss-Newton, os valores de $\Delta a, \Delta b_{1}$ e $\Delta b_{2}$. Se essas correções não forem desprezíveis, obtemos $a_{1}={ }_{0} a+\Delta a, b_{1}={ }_{0} b_{1}+\Delta b_{1} e b_{2}={ }_{0} b_{2}+\Delta b_{2}$. A seguir, utilizando $a_{1}, b_{1}$ e $b_{2}$ como novas estimativas preliminares, os cálculos são refeitos, obtendo-se novas correções $\Delta a, \Delta b_{1}$ e $\Delta b_{2}$.

Admitindo-se que o processo seja convergente, isto ë, que os valores absolutos das correções tendam a zero, os cálculos são repetidos até que as correções $\Delta a, \Delta b_{1}$ e $\Delta b_{2}$ sejam consideradas desprezíveis. Chega-se, assim, às estimativas de quadrados mínimos $\underline{a}, \underline{b}_{1}$ e $\underline{b}_{2}$ dos parámetros $A, B_{1}$ e $B_{2}$.

Cälculo das estimativas preliminares $0_{a_{0}} o_{1} e_{0} b_{2}$

$$
\text { a método usado na obtenção das estimativas iniciais }
$$

foi o seguinte.

Consideremos,

$$
\begin{gathered}
Y_{i}=A X_{11}^{B_{1}} X_{i 2}^{B_{2}}\left(1+\varepsilon_{i}\right)= \\
\therefore \quad L Y_{i}=L A+B_{1}\left(L X_{i 1}\right)+B_{2}\left(L X_{i 2}\right)+L\left(1+\varepsilon_{i}\right)
\end{gathered}
$$

fazendo,

$$
\begin{aligned}
y_{i} & =L Y_{i} \\
a & =L A \\
x_{i 1} & =L X_{i 1} \\
x_{i 2} & =L X_{i 2} \\
e_{i} & =L\left(1+\varepsilon_{i}\right)
\end{aligned}
$$


temos,

$$
y_{i}=a+\theta_{1} x_{i_{1}}+\theta_{2} x_{i_{2}}+e_{i} \text {. }
$$

que é uma equação de regressão linear múltipla, cuja aplicação é bem conhecida.

Destra forma, obtivemos as estimativas preliminares oa $0 \underline{b}_{1},{ }_{\theta} \underline{b}_{2}$, dadas por $\tilde{a}, \hat{B}_{1}, \hat{B}_{2}$, através das relações:

$$
\begin{aligned}
& { }_{0}^{a}=\exp \hat{a}= \\
& { }_{0} b_{1}=\hat{B}_{1}, \\
& { }_{0} b_{2}=\hat{E}_{2} .
\end{aligned}
$$

\subsubsection{3 - Generaljzação do processo}

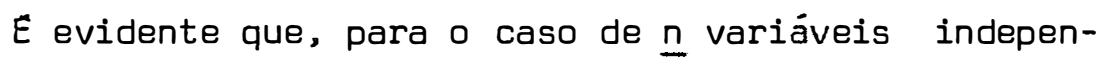

dentes, isto é,

$$
y_{i}=A x_{i 1}^{B_{1}} x_{i 2}^{B_{2}} \ldots x_{i n}^{B_{n}}+e_{i},
$$

ou

$$
y_{i}=A \prod_{k=1}^{n} x_{i k}^{B_{k}}+e_{i},
$$

com $i=1,2, \ldots, N, X_{i j}>0$ para $j=1,2, \ldots, n, A>0, \theta_{j}>0$

\section{Se fizermos}

$$
\begin{aligned}
& f_{n}=x_{i 1}^{2}{ }_{0}^{b} x_{i 2}{ }^{2} b_{2} b_{2} \ldots x_{i n}^{2} b_{n}^{b}, g_{n}=\left(y_{i}-y_{i}^{*}\right) x_{i 1}^{b_{1}^{b}} x_{i 2}^{b^{b}} \ldots x_{i n}^{b^{b}}, \\
& \ell_{1}=L x_{11}, \quad \ell_{2}=L x_{i 2}, \ldots, \ell_{n}=L x_{i n},
\end{aligned}
$$


onde $a_{0},{ }_{0} b_{1},{ }_{0} b_{2}, \cdots, b_{n}^{b}$ são estimativas preliminares e

$$
y_{i}^{*}=a_{0} x_{i 1}^{0^{b_{1}}} x_{i 2}^{0^{b_{2}}} \ldots x_{i n}^{0^{b} n} \text {, }
$$

temos:

$$
\left[\begin{array}{c}
\Delta a \\
\Delta b_{1} \\
\Delta b_{2} \\
. \\
\Delta b_{n}
\end{array}\right]=[\Psi]^{-1}\left[\begin{array}{c}
\sum g_{n} \\
\Sigma g_{n} l_{1} \\
\Sigma g_{n} l_{2} \\
\cdots \\
\Sigma g_{n} l_{n}
\end{array}\right],
$$

onde, para o método de Newton, 


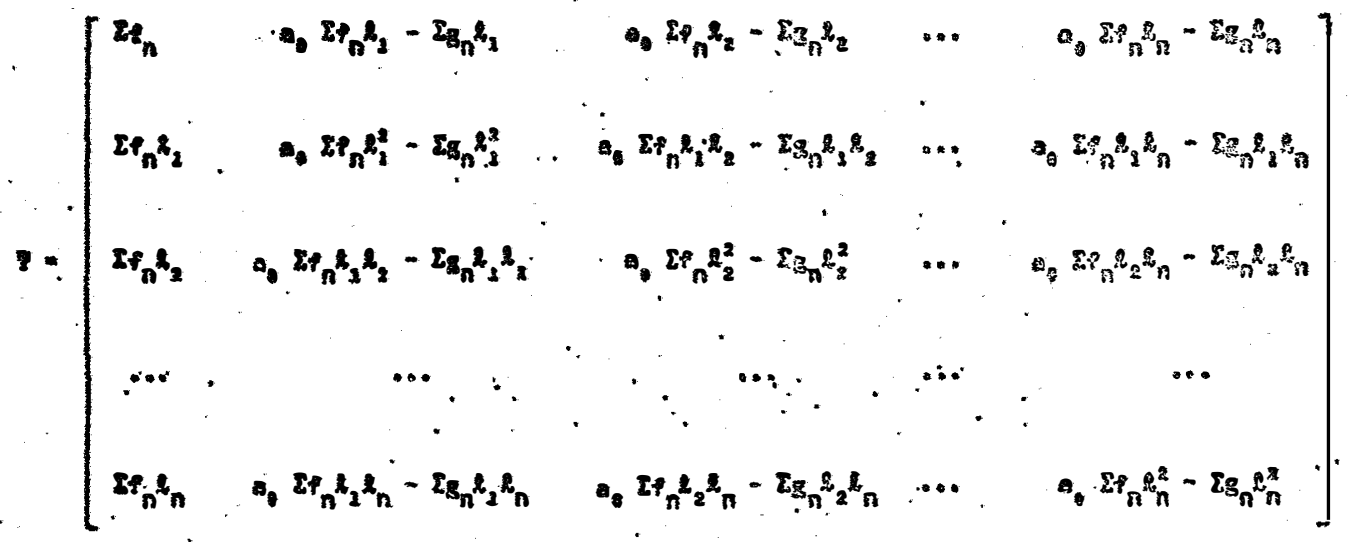

8. para o método modıf 1cado de Gouss-Newton,

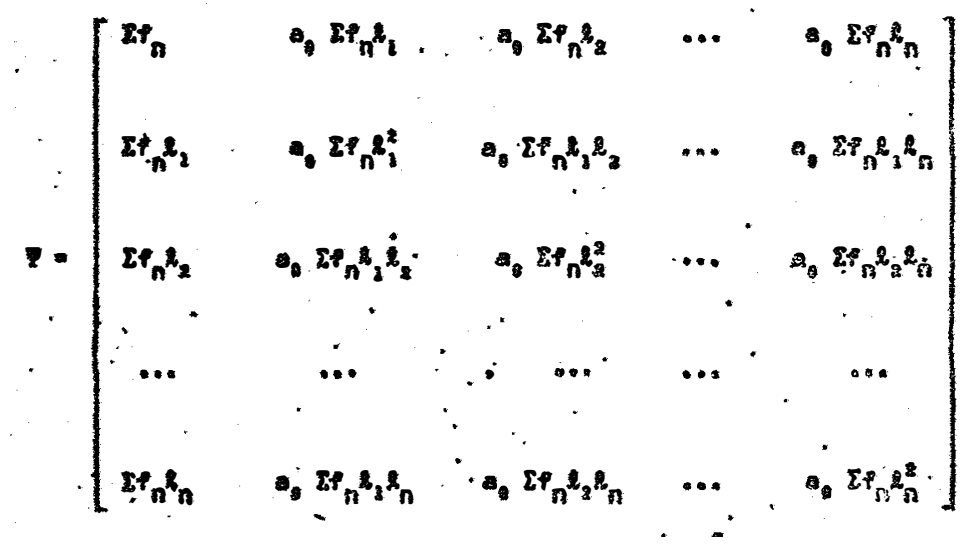


As estimativas preliminares seriam obtidas da seguin

te forma:

$$
\begin{gathered}
Y_{i}=A X_{i 1}^{B_{1}} X_{i 2}^{B_{2}} \ldots X_{i n}^{B_{n}}\left(1+\varepsilon_{i}\right) \\
L Y_{i}=L A+B_{1}\left(L X_{i 1}\right)+B_{2}\left(L X_{i 2}\right)+\ldots+B_{n} L\left(X_{i n}\right)+L\left(1+\varepsilon_{i}\right) 。
\end{gathered}
$$

Fazendo

$$
\begin{aligned}
y_{i} & =L Y_{i} \\
\hat{a} & =L A \\
x_{i 1} & =L x_{i 1} \\
x_{i 2} & =L x_{i 2} \\
\ldots & \ldots \\
x_{i n} & =L X_{i n} \\
e_{i} & =L\left(I+\varepsilon_{i}\right),
\end{aligned}
$$

temos.

$$
y_{i}=a+B_{1} x_{i 1}+B_{2} x_{i 2}+\ldots+B_{n} x_{i n}+e_{i},
$$

que é uma equação de regressão linear múltipla, cuja aplicação é bem conhecida.

Desta forma, obtêm-se as estimativas preliminares 0 a, $0 \underline{b}_{1}, b_{2}, \ldots, \underline{b}_{n}$, dadas por $\hat{a}_{1} \hat{\mathrm{B}}_{1}, \hat{\mathrm{B}}_{2}, \ldots, \hat{\mathrm{B}}_{\mathrm{n}}$, através das relações:

$$
\begin{aligned}
& o^{a}=\exp \hat{a}, \\
& { }_{0} b_{k}=\tilde{B}_{k},
\end{aligned}
$$

$\operatorname{com} k=1,2, \ldots, n$. 


\subsubsection{4 - Estimativa da dose econômica}

Caso de uma sö variävel independente

$$
\begin{aligned}
& \text { Seja, } \bar{\gamma}_{i}=a X_{i}^{b}\left(a, b>0, x_{i}>0\right) . \\
& \text { A condição de maximização dos lucros ou da receita }
\end{aligned}
$$
líquida é que a receita marginal seja igual ao custo marginal, desde que estejam satisfeitas as condições suficientes relativas à diferencial segunda da receita líquida, isto é, $0<b<1$, no caso presente.

Consideremos,

$$
\begin{aligned}
P_{X} & =\text { preço de } X \text { (nutriente }) \\
P_{Y} & =\text { preço de } Y(\text { produto }) \\
R T & =\text { receita total: } \\
R M g & =\text { receita marginal: } \\
C T & =\text { custo total: } \\
\mathrm{CMg} & =\text { custo marginal. }
\end{aligned}
$$

Sabemos que:

$$
\begin{gathered}
R T=P_{Y} \cdot P ; R T=P_{Y} \circ a X^{b} ; \quad R M g=\frac{d R T}{d X}=a b X^{b-1} P_{Y}, \\
C T=P_{X} X ; C M g=\frac{d C T}{d X}=P_{X} .
\end{gathered}
$$

Portanto, para $0<b<1$ temos:

$$
a b X^{b-1} P_{Y}=P_{X}
$$


35.

$$
\begin{aligned}
X^{b-1} & =\frac{P_{X}}{P_{Y}} \cdot \frac{1}{a b} . \\
X^{*} & =\left(\frac{P_{X}}{P_{Y}} \cdot \frac{1}{a b}\right)^{1 /(b-1)}
\end{aligned}
$$

Sendo RL a receita líquida, temos

$$
R L=P_{Y} a X^{b}-P_{X} X
$$

$\log 0$,

$$
d^{2} R L=P_{Y} b(b-1) a X^{b-2} d x^{2}
$$

Conclui-se, pois, que $d^{2} R L$ é negativo para $0<b<1$, como se afirmou.

Caso de duas variäveis independentes

$$
\text { Seja, } \gamma=a x_{1}^{b_{1}} x_{2}^{b_{2}}\left(a, b_{1}, b_{2}>0, x_{1}, x_{2}>0\right), e
$$

consideremos,

$$
\begin{aligned}
& P_{X_{1}}=\text { preço de } X_{1} \text { (nutriente); } \\
& P_{X_{2}}=\text { preço de } X_{2} \text { (nutriente); } \\
& P_{Y}=\text { preço de } Y \text { (produto). }
\end{aligned}
$$

Portanto:

$$
R T=P_{Y} 8 ; \quad R T=P_{Y} a X_{1}^{b_{1}} x_{2}^{b_{2}} ; C T=P_{X_{1}} X_{1}+P_{X_{2}} X_{2} .
$$

A condição de lucro máximo é: 


$$
R M g_{X_{1}}=C M g_{X_{1}}, \quad R M g_{X_{2}}=\mathrm{CMg}_{X_{2}}
$$

onde, $\mathrm{RMg}_{\mathrm{X}_{1}}=$ receita marginal de $\mathrm{X}_{1}$,

$$
\begin{aligned}
& \mathrm{RMg}_{\mathrm{X}_{2}}=\text { receita marginal de } \mathrm{X}_{2}, \\
& \mathrm{CMg}_{\mathrm{X}_{1}}=\text { custo marginal de } \mathrm{X}_{1}, \\
& \mathrm{CMg}_{\mathrm{X}_{2}}=\text { custo marginal de } \mathrm{X}_{2},
\end{aligned}
$$

desde que estejam satisfeitas as condições suficientes relativas à diferencial segunda da receita líquida.

Mas,

$$
\begin{aligned}
& R M g_{X_{1}}=\frac{\partial}{\partial X_{1}} R T=P_{Y} a b_{1} X_{1}^{b_{1}^{-1} X_{2}^{b_{2},},} \\
& \operatorname{RMg}_{X_{2}}=\frac{\partial}{\partial X_{2}} R T=P_{Y} a b_{2} X_{1}^{b_{1} X_{2}^{b_{2}-1},} \\
& \operatorname{CPIg}_{X_{1}}=\frac{\partial}{\partial X_{1}} C T=P_{X_{1}}, \\
& \mathrm{CMg}_{X_{2}}=\frac{\partial}{\partial X_{2}} C T=P_{X_{2}},
\end{aligned}
$$

então

$$
\left\{\begin{aligned}
P_{Y} a b_{1} x_{1}^{b_{1}-1} x_{2}^{b_{2}} & =P_{X_{1}} \\
P_{Y} a b_{2} X_{1}^{b_{1}} x_{2}^{b_{2}-1} & =P_{X_{2}}
\end{aligned}\right.
$$

cuja solução conduz às raf́zes $X_{1}^{*}$ e $X_{2}^{*}$ que maximizam a receita líqui da, desde que estejam satisfeitas as condições suficientes relativas à diferencial segunda da receita líquida. 
Caso de $n$ variäveis independentes

$$
\text { Seja, } q=a x_{1}^{b_{1}} x_{2}^{b_{2}} \ldots x_{n}^{b_{n}} \text {, então }(\zeta) \text { assumirá a }
$$

forma

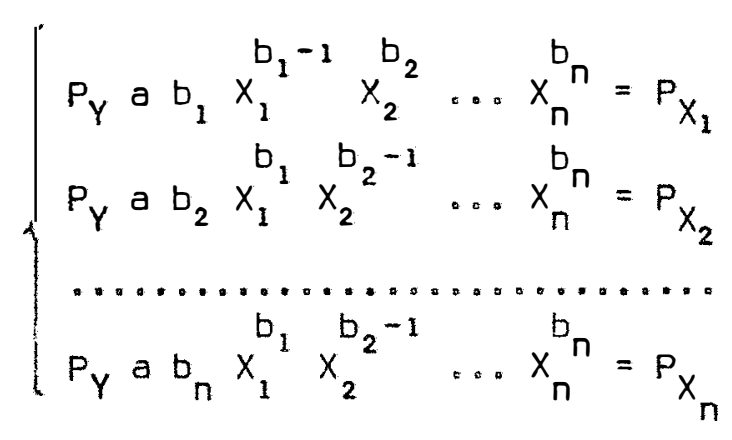

cuja solução conduz às raízes $X_{1}^{*}, X_{2}^{*}, \ldots, x_{n}^{*}$ que maximizam a receita líquida, desde que estejam satisfeitas as condições suficientes re lativas à diferencial segunda da receita líquida 
38.

\section{RESULTADOS E DISCUSSÃO}

\section{1 - Modelos com Erro Multiplicativo}

0 Quadro 1 mostra a análise de variância dos dados do primeiro ensaio pelo método do erro multiplicativo, cuja equação de regressão ë

$$
P=103,5268 \times 0,3705,
$$

e o Quadro 2 apresenta a análise de variància dos dados do segundo ensaio, nas mesmas condições anteriores, e cuja equação de regręsão estimada é

$$
P=70,9307 \times 11957 \times \frac{0,3461}{0.195} .
$$


Quadro 1 - Análise de variância relativa ao ensaio $n^{*} l$.

\begin{tabular}{lrrrr}
\hline Causa da Variação & G.L. & S.Q. & Q.M. & F \\
\hline Regressão & 1 & 26934,1767 & 26934,1767 & 5783,96 ** \\
Desvios da Regressão & 10 & 4073,7000 & 407,3700 & 87,48 ** \\
\hline (Tratamentos) & $(11)$ & $(31007,8767)$ & & \\
Resíduo & 36 & 167,6400 & 4,6567 & \\
\hline Total & 47 & 31175,5167 & & \\
\hline
\end{tabular}

Quadro 2 - Análise de variância relativa ao ensaio $n^{8} 2$.

\begin{tabular}{lccrr}
\hline Causa da Variação & G.L. & S.Q. & Q.M. & F \\
\hline Regressão & 2 & 34091,7347 & 17045,8674 & 7801,67 ** \\
Desvios da Regressão & 9 & 40,9976 & 4,5553 & 2,08 ns \\
(Tratamentos) & $(11)$ & $(34132,7323)$ & \\
ResIduo & 36 & 78,6575 & 2,1849 & \\
\hline Total & 47 & 34211,3898 & & \\
\hline
\end{tabular}

Observa-se no Quadro 1 efeito altamente significativo, isto $\bar{e}$, significativo ao nível de $1 \%$ de probabilidade, tanto pa ra a regressão como para os desvios de regressão. Entretanto,no Qua dro 2 enquanto a regressão foi altamente significativa os desvios de regressão não o foram. 
As estimativas dos parâmetros assim obtidas foramusa das como valores iniciais nos métodos iterativos aplicados a seguir.

\section{2 - Modelo 1 - Com Erro Aditivo}

Após quatro iterações, para o método de Newton e cin co para o método modificado de Gauss-Newton, com valores iniciais $a_{0}=103,5268, b_{0}=0,3705$, obtiveram-se as seguintes estimativas pe lo método de Newton:

$$
\begin{aligned}
& a=104,8619 \\
& b=0,3248 .
\end{aligned}
$$

Temos, pois, a equação estimada:

$$
\varphi=104,0619 \times 19,3248
$$

Com os mesmos valores iniciais, o método modificado de Gauss-Newton deu:

$$
\begin{aligned}
& a=104,862, \\
& b=0,3248,
\end{aligned}
$$

cuja equação correspondente é:

$$
P=104,862 \times 0,3248
$$

Os Quadros 3 e 4 mostram as correçöes obtidas com as iterações, quando se aplicaram esses métodos. Deles se depreende que, 
com a aplicação do método de Newton, a função ajustada convergiu mais rapidamente.

Quadro 3 - Correções correspondentes às iterações do modelo I pelo método de Newton.

\begin{tabular}{ccccc}
\hline \multirow{2}{*}{ CORREÇÕES } & \multicolumn{4}{c}{ ITERAÇÕES } \\
\cline { 2 - 5 } & $\mathbf{l}^{\mathrm{a}}$ & $2 !$ & $3 !$ & $4 !$ \\
\hline$\Delta a$ & 5,9731 & $-0,6226$ & $-0,0103$ & 0,000016 \\
$\Delta b$ & $-0,0515$ & 0,00560 & 0,00023 & $-0,000033$ \\
\hline
\end{tabular}

Quadro 4 - Correções correspondentes às iterações do modelo I pelo método modificado de Gauss-Newton.

\begin{tabular}{cccccc}
\hline \multirow{2}{*}{ CORREÇÕES } & \multicolumn{5}{c}{ ITERAÇÕES } \\
\cline { 2 - 6 } & $1^{a}$ & $2^{a}$ & $3 !$ & $4 !$ & $5 !$ \\
\hline$\Delta a$ & 6,1111 & $-0,8316$ & 0,0725 & $-0,0136$ & 0,0022 \\
$\Delta b$ & $-0,0515$ & 0,0068 & $-0,00120$ & 0,00021 & $-0,000042$ \\
\hline
\end{tabular}

Em vifthde dos ajustamentos oriundos da aplicação do método de Newton e do método modificado de Gauss-Newton terem convergido para uma mesma função, isto é, para

$$
8=104,862 \times 03248,
$$

apresenta-se, no Quadro 5, uma só análise de variāncia. 
Quadro 5 - Análise de variáncia relativa ao ensaio $n^{8} 1$.

\begin{tabular}{lcrrr}
\hline Causa da Variação & G.L. & S.Q. & Q.M. & \multicolumn{1}{c}{ F } \\
\hline Regressão & 1 & 27351,2295 & 27351,2295 & $5873,52 * *$ \\
Desvios da Regressão & 10 & 3656,6472 & 365,6647 & $73,52 * *$ \\
(Tratamentos) & $(11)$ & $(31007,3767)$ & & \\
Resíduo & 36 & 167,6400 & 4,6567 & \\
\hline Total & 47 & 31175,5167 & & \\
\hline
\end{tabular}

Observa-se no Quadro 5 efeito altamente significativo, ao nỉvel de $1 \%$ de probabilidade, tanto para a regressão, como para os desvios da regressão. Entretanto, a soma de quadrados dos desvios de regressão foi menor que a obtida quando se usou o erro multiplicativo, isto sugerindo um melhor ajustamento no caso do erro aditivo, embora, em ambos, o quadrado médio dos desvios da regressão tenha sido significativo ao nível de $1 \%$ de probabilidade. 
Quadro 6 - Demonstrativo do comportamento das funções ajustadas do modelo I.

\begin{tabular}{rrrrr}
\hline$X$ & $\bar{Y}$ & $\bar{Y}=103,5268 \times X^{0,3705}$ & $(\bar{Y}-\bar{Y})$ & $(\bar{Y}-P)^{2}$ \\
\hline 0,2 & 53,60 & 57,0275 & $-3,4275$ & 11,7478 \\
0,4 & 69,90 & 73,7252 & $-3,8252$ & 14,6322 \\
0,6 & 86,20 & 85,6757 & 0,5243 & 0,2749 \\
0,8 & 101,00 & 95,3120 & 5,6880 & 32,3533 \\
1,0 & 110,00 & 103,5268 & 6,4732 & 41,9023 \\
1,2 & 119,00 & 110,7617 & 8,2383 & 67,8696 \\
1,4 & 128,00 & 117,2717 & 10,7283 & 115,0964 \\
1,6 & 132,00 & 123,2195 & 8,7805 & 77,0972 \\
1,8 & 133,00 & 128,7156 & 4,2844 & 18,3561 \\
2,0 & 131,00 & 133,8395 & $-2,8395$ & 8,0628 \\
2,2 & 129,00 & 138,6502 & $-9,6502$ & 93,1264 \\
2,4 & 120,00 & 143,1928 & $-23,1928$ & 537,9060 \\
\hline
\end{tabular}

\begin{tabular}{rrrrr}
\hline$X$ & $\bar{Y}$ & $P_{1}=104,862 \times x^{0,3248}$ & $\left(\bar{Y}-P_{1}\right)$ & $\left(\bar{Y}-P_{1}\right)^{2}$ \\
\hline 0,2 & 53,60 & 62,1716 & $-8,5716$ & 73,4723 \\
0,4 & 69,90 & 77,8694 & $-7,9694$ & 63,5113 \\
0,6 & 86,20 & 88,8303 & $-2,6303$ & 6,9185 \\
0,8 & 101,00 & 97,5307 & 3,4693 & 12,0360 \\
1,0 & 110,00 & 104,8619 & 5,1381 & 26,4001 \\
1,2 & 119,00 & 111,2591 & 7,7409 & 59,9215 \\
1,4 & 128,00 & 116,9715 & 11,0285 & 121,6278 \\
1,6 & 132,00 & 122,1563 & 9,8437 & 96,8984 \\
1,8 & 133,00 & 126,9200 & 6,0800 & 36,9664 \\
2,0 & 131,00 & 131,3385 & $-0,3385$ & 0,1146 \\
2,2 & 129,00 & 135,4679 & $-6,4679$ & 41,8337 \\
2,4 & 120,00 & 139,3510 & $-19,3510$ & 374,4612 \\
\hline
\end{tabular}




\section{3 - Modelo 11 - Com Erro Aditivo}

Após cinco iterações, para o método de Newton, com valores iniciais $0^{a}=70,9307,{ }_{0}^{b}{ }_{1}=0,1957,{ }_{0}^{b}{ }_{2}=0,3461$, observou-se não haver convergência das estimativas dos parâmetros. Porém, para o método modificado de Gauss-Newton, e com os mesmos valores iniciais, fizeram-se três iterações e se obtiveram as seguintes estimativas:

$$
\begin{aligned}
& a=70,5766, \\
& b_{1}=0,1749, \\
& b_{2}=0,3717 .
\end{aligned}
$$

Temos, pois, a equação estimada:

$$
8=70,5766 \times 10,1749 \times 0,3717
$$

o fato de não ter havido convergência para as estima tivas dos parâmetros quando se aplicou o método de Newton, impossibilita uma comparação, nesse caso, com o método tradicional, isto é, aquele que usa o erro aleatório multiplicativo.

A explicação para a falta de convergência, dada pelos estudiosos do assunto, é o fato de as estimativas iniciais não terem sido adequadas. Mas este é, sem dúvida, o defeito mais sério dos métodos iterativos mencionados.

Os Quadros 7 e 8 mostram as correções que foram obti das para o método de Newton e para o método modificado de Gauss-New 
ton, respectivamente.

Quadro 7 - Correções correspondentes a 1terações do modelo II pela aplicação do método de Newton.

\begin{tabular}{cccccc}
\hline \multirow{2}{*}{ CORREÇÕES } & \multicolumn{5}{c}{ ITERAÇÕES } \\
\cline { 2 - 6 } & $1_{0}^{a}$ & $2^{a}$ & $3 \cdot$ & $4 !$ & 5. \\
\hline$\Delta a$ & $0,7.10$ & 1,6792 & $-5,1506$ & $-0,0992$ & 1,5995 \\
$\Delta b_{1}$ & 0,030 & 0,0421 & $-0,1274$ & 0,0115 & 0,0264 \\
$\Delta b_{2}$ & $-0,0257$ & $-0,0442$ & 0,1376 & $-0,0097$ & $-0,0332$ \\
\hline
\end{tabular}

Quadro 8 - Correções correspondentes a iterações do modelo II pela aplicação do método modificado de Gauss-Newton.

\begin{tabular}{cccc}
\hline \multirow{2}{*}{ CORREÇÕES } & \multicolumn{3}{c}{ ITERAÇÕES } \\
\cline { 2 - 4 } & $1_{0}^{\mathrm{a}}$ & $2 !$ & $3 \stackrel{\mathrm{a}}{\mathrm{a}}$ \\
\hline$\Delta \mathrm{a}$ & $-1,4236$ & 0,0071 & $-0,000067$ \\
$\Delta \mathrm{b}_{1}$ & $-0,0209$ & 0,000084 & $-0,000040$ \\
$\Delta \mathrm{b}_{2}$ & 0,0259 & $-0,00030$ & $-0,000020$ \\
\hline
\end{tabular}

0 Quadro 9 mostra a anälise de variância para o mode lo II da regressão, após as iterações, ao aplicar-se o método mod1ficado de Gauss-Newton. 
Quadro 9 - Anälise de variância relativa ao ensaio $n^{8} 2$.

\begin{tabular}{lrrrr}
\hline Causa da Variação & G.L. & \multicolumn{1}{c}{ S.Q. } & Q.M. & \multicolumn{1}{c}{ F } \\
\hline Regressão & 2 & 34092,6287 & 17046,3144 & 7801,87 ** \\
Desvios de Regressão & 9 & 40,1036 & 4,4560 & $2,04 \mathrm{~ns}$ \\
\hline (Tratamentos) & $(11)$ & $(34132,7323)$ & & \\
Resíduo & 36 & 78,6575 & 2,1849 & \\
\hline Total & 47 & 34211,3898 & & \\
\hline
\end{tabular}

Observa-se que a soma de quadrados dos desvios de re gressão para o método modificado de Gauss-Newton foi ligeiramente menor que a soma de quadrados dos desvios de regressão para o caso em que se adotou o erro aleatório multiplicativo. 
Quadro 10 - Demonstrativo do comportamento das funções ajustadas do modelo II.

\begin{tabular}{rrrrrrr}
\hline$X_{1}$ & $X_{2}$ & $\bar{Y}$ & $P=70,93068 X_{1}^{0,2957} X_{2}^{0,3461}$ & $(\bar{Y}-\bar{Y})$ & $(\bar{Y}-P)^{2}$ \\
\hline 0,1 & 0,2 & 25,650 & 25,8953 & $-0,2453$ & 0,0602 \\
0,3 & 0,4 & 41,400 & 40,8112 & 0,5888 & 0,3467 \\
0,5 & 0,6 & 52,200 & 51,8968 & 0,3032 & 0,0919 \\
0,7 & 0,8 & 60,275 & 61,2321 & $-0,9571$ & 0,9160 \\
0,9 & 1,0 & 68,975 & 69,4831 & $-0,5081$ & 0,2582 \\
1,1 & 1,2 & 76,950 & 76,9732 & $-0,0232$ & 0,0005 \\
1,3 & 1,4 & 83,150 & 83,8895 & $-0,7395$ & 0,5469 \\
1,5 & 1,6 & 89,725 & 90,3527 & $-0,6277$ & 0,3940 \\
1,7 & 1,8 & 96,625 & 96,4457 & 0,1793 & 0,3321 \\
1,9 & 2,0 & 102,675 & 102,2287 & 0,4464 & 0,1993 \\
2,1 & 2,2 & 109,800 & 107,7469 & 2,0531 & 4,2152 \\
2,3 & 2,4 & 111,250 & 113,0356 & $-1,7856$ & 3,1884 \\
\hline
\end{tabular}

\begin{tabular}{rrrrrrr}
\hline$X_{1}$ & $X_{2}$ & $\bar{Y}$ & $P_{1}=70,5766 X_{1}^{0,1749} X_{2}^{0,3717}$ & $\left(\bar{Y}-P_{1}\right)\left(\bar{Y}-P_{1}\right)^{2}$ \\
\hline 0,1 & 0,2 & 25,650 & 25,9390 & $-0,2890$ & 0,0835 \\
0,3 & 0,4 & 41,400 & 40,6719 & 0,7281 & 0,5301 \\
0,5 & 0,6 & 52,200 & 51,7070 & 0,4930 & 0,2431 \\
0,7 & 0,8 & 60,275 & 61,0305 & $-0,7555$ & 0,5707 \\
0,9 & 1,0 & 68,975 & 69,2879 & $-0,3129$ & 0,0979 \\
1,1 & 1,2 & 76,950 & 76,7948 & 0,1552 & 0,0241 \\
1,3 & 1,4 & 83,150 & 83,7346 & $-0,5846$ & 0,3418 \\
1,5 & 1,6 & 89,725 & 90,2257 & $-0,5007$ & 0,2507 \\
1,7 & 1,8 & 96,625 & 96,3498 & 0,2752 & 0,0757 \\
1,9 & 2,0 & 102,675 & 102,1662 & 0,5088 & 0,2589 \\
2,1 & 2,2 & 109,800 & 107,7197 & 2,0803 & 4,3276 \\
2,3 & 2,4 & 111,250 & 113,0450 & $-1,7950$ & 3,2218 \\
\hline & \multicolumn{7}{c}{} & & 10,0259 \\
\hline
\end{tabular}


48.

\section{CONCLUSÖES}

5.1 - Para o primeiro modelo ajustado aos dados do ensaio $n^{8} 1$, conclui-se que o erro aleatório aditivo apresentou um melhor ajustamento que o tradicional de erro aleatório multiplicativo, tanto com o uso do método de Newton como para o caso do método modificado de Gauss-Newton.

5.2 - Para o segundo modelo ajustado aos dados do ensaio $n^{9} 2$, conclui-se que o método que propuzemos apresentou um melhor ajusta mento que o tradicional, apenas com o uso do método modificado de Gauss-Newton. A comparação com o método de Newton não foi possível em virtude de, na sua aplicação não ter ocorrido convergência, para as estimativas dos parâmetros.

5.3 - Nos casos de convergência, esta ocorreu com bastante rapi dez, de tal sorte que poucas iterações foram necessárias. 


\section{BIBLIOGRAFIA}

CAMARGO, J.R.V. de, 1974. Análise da Produtividade nas Culturas de Algodão e Soja com a Aplicação do Modelo Ulveling-Fletcher. Piracicaba, ESALQ/USP. 131 pp. (Dissertação de Mestrado).

COSTA LIMA, A.R., 1980. Superfícies de Resposta em Experimentos Fa toriais $3^{3}$ Incompletos de Adubação NPK em Mandioca no Estado do Ceará. Piracicaba, ESALQ/USP. 100 pp. (Dissertação de Mestrado).

CRócomo, D.H.G., 1974. Oferta de Milho e de Soja. Uma Análise a Par tir de Funções de Produção. Piracicaba, ESALQ/USP. 94 pp. (Dis sertação de Mestrado\}.

OIXON, J.W. e F.J. MASSEY Jr., 1951. Introduction to Statistical. Analysis. Nova York, McGraw-Hill, 370 pp. 
ENGLER, J.J. de C., 1978. Análise da Produtividade Agrícola Entre Regiões do Estado de São Paulo. Piracicaba, ESALq/USP. 132 pp. (Tese de Livre Docência).

ENGLER, J.J. de C., 1968. Análise da Produtividade de Recursos na Agricultura. Piracicaba, ESALQ/USP. 102 pp. (Tese de toutoramento).

GIRÃo, J.A., 1965. A Função de Produção de Cobb-Douglas e a Análi-. se Inter-Regional da Produção Agrícnla. Lisboa, Fundação Calous te Gulbenkian, Centro de Estudos de Economia Agrária, 119 pp.

HARTLEY, H.D., 1961. The Modified Gauss-Newton Method for the Fitting of Non-Linear Regression, Functions by Least Squares. Technometrics, 3: 269-280.

HOFFMANN, R. e S. VIEIRA, 1977. Anälise de Regressão. Uma Introdução à Econometria. São Paulo. HIJCITEC,-EDUSP, 339 pp.

MALINVAUD, E。, 1969. Methodes Statistiques de L'Econometrie. Paris, Dunod, $645 \mathrm{pp}$.

MELO, F.I.0., 1976. Aplicação do Método Modificado de Gauss-Newton para Estimar os Parâmetros da Equação de Mitscherlich. Piracica ba, ESALQ/USP. 74 pp. (Dissertação de Mestrado).

MITSCHERLICH, E.A., 1909. Das Gesetz des Minimums und das Gesetz des Abnehmenden Bodenertrages. Landwirtschaftliche Jahrbücher, Berlim, 38: 537-552.

NEVES, E.M., 1972. Uma Função de Produção de Leite no Estado de São Paulo. Piracicaba, ESALQ/USP. 72 pp. (Tese de Doutoramento). 
NOJIMOTO, T., 1976. Problemas Encontrados na Estimação e Interpretação de Funções de Produção Agrícola. Piracicaba, ESALß̧/USP. 118 pp. (Dissertação de Mestrado).

PERRE DA SILVA, M.A。, 1978. Segunda Aproximação de Mitscherlich, $Y=A\left[1-10^{-c(x+b)}\right] 10^{-k(x+b)^{2}}$ Aplicada à Adubação Mineral. P $\underline{\underline{i}}$ racicaba, ESALQ/USP. 96 pp. (Dissertação de Mestrado).

PIMENTEL GOMES, F。 e A.G. ZAGATTO, 1967. Aspectos Económicos da Adubação. In: MALAVOLTA, E. Manual de Química Agrícola - Adubos. e Adubação. São Paulo. Agr. Ceres, 606 pp.

PINHEIRO, F.A., 1972. Análise Econométrica de Alocação de Recursos na Produção Bovina do Município de Botucatu. Piracicaba, ESALQ/ USP. (Dissertação de Mestrado).

SILVA, Z.P., 1973. Uso e Eficiência do Crédito Rural e dos Fatores de Produção. Piracicaba, ESALQ/USP. (Dissertação de Mestrado).

SILVEIRA JUNIOR, P., 1976. Estudo de Alguns Modelos Exponenciais no Crescimento de Bovinos de Raça Ibagé. Piracicaba, ESALQ/USP, 174 pp. (Dissertação de Mestrado).

SIMONSEN, M.H., 1971. Teoria Microeconômica, 4! edição, Rio de Ja neiro, Fundação Getūlio Vargas (Vol. 1). 425 pp. 
52.

7. APENDICE 
Tabela 1 - Ensaio $n^{8} 1$ - Dados obtidos por simulação, com média $m=$ $=100$ e variância $\sigma^{2}=12$, por PERRE DA SILVA (1978).

\begin{tabular}{cccccc}
\hline$x$ & \multicolumn{4}{c}{ REPET IÇÕES } & TOTAIS \\
\cline { 2 - 5 } & 10 & $2 !$ & 30 & $4 !$ & \\
\hline 0,2 & 52,8 & 50,4 & 55,0 & 56,2 & 214,4 \\
0,4 & 70,0 & 68,4 & 65,2 & 75,0 & 279,6 \\
0,6 & 88,0 & 87,0 & 85,8 & 84,0 & 344,0 \\
0,8 & 103,0 & 101,0 & 100,8 & 99,2 & 404,0 \\
1,0 & 109,4 & 111,0 & 110,0 & 109,6 & 440,0 \\
1,2 & 120,0 & 119,8 & 121,0 & 115,2 & 476,0 \\
1,4 & 128,0 & 129,0 & 127,0 & 128,0 & 512,0 \\
1,6 & 133,4 & 132,6 & 131,0 & 131,0 & 528,0 \\
1,8 & 135,0 & 132,0 & 134,0 & 131,0 & 532,0 \\
2,0 & 132,0 & 134,0 & 130,0 & 128,0 & 524,0 \\
2,2 & 130,0 & 127,0 & 130,0 & 129,0 & 516,0 \\
2,4 & 119,0 & 117,0 & 124,0 & 120,0 & 480,0 \\
\hline
\end{tabular}


Tabela 2 - Ensaio $n^{8} 2$ - Dados obtidos por simulação, pelo modelo $Y=68,2857 x_{i 1}^{0,2168} x_{i 2}^{0,3542}+e_{i}, \operatorname{com} e_{i} \cap N(0,4)$.

\begin{tabular}{|c|c|c|c|c|c|c|}
\hline \multirow{2}{*}{$x_{1}$} & \multirow{2}{*}{$x_{2}$} & \multicolumn{4}{|c|}{ REPET IÇÕES } & \multirow{2}{*}{ TOTAIS } \\
\hline & & 1 a & $2^{a}$ & $3^{\mathrm{a}}$ & $4 \stackrel{a}{a}$ & \\
\hline 0,1 & 0,2 & 23,5 & 27,1 & 27,0 & 25,0 & 102,6 \\
\hline 0,3 & 0,4 & 40,9 & 42,2 & 42,3 & 40,2 & 165,6 \\
\hline 0,5 & 0,6 & 50,7 & 52,0 & 52,4 & 53,7 & 208,8 \\
\hline 0,7 & 0,8 & 59,5 & 60,0 & 60,7 & 60,9 & 241,1 \\
\hline 0,9 & 1,0 & 68,6 & 70,4 & 68,9 & 68,0 & 275,9 \\
\hline 1,1 & 1,2 & 79,0 & 74,6 & 78,5 & 75,7 & 307,8 \\
\hline 1,3 & 1,4 & 82,0 & 84,4 & 84,3 & 81,9 & 332,6 \\
\hline 1,5 & 1,6 & 89,3 & 91,0 & 90,2 & 88,4 & 358,9 \\
\hline 1,7 & 1,8 & 96,3 & 95,6 & 99,0 & 95,6 & 386,5 \\
\hline 1,9 & 2,0 & 102,5 & 100,4 & 102,8 & $.105,0$ & 410,7 \\
\hline 2,1 & 2,2 & 111,0 & 110,2 & 107,3 & 110,7 & 439,2 \\
\hline 2,3 & 2,4 & 112,5 & 112,6 & 114,1 & 115,8 & 445,0 \\
\hline
\end{tabular}


55.

Tabela 3 - Valores iniciais utilizados para o ajustamento dos modelos I e II aos totais de tratamentos, com 4 repetições.

\begin{tabular}{|c|c|c|c|}
\hline \multirow{2}{*}{ MODELO } & \multicolumn{3}{|c|}{ VALORES INICIAIS } \\
\hline & $a_{0}$ & ${ }_{0} b_{1}$ & ${ }_{0} b_{2}$ \\
\hline$y_{i}=A X_{i}^{B}+e_{i}$ & 414,1072 & 0,3705 & $\cdots$ \\
\hline$Y_{i}=A X_{i 1}^{B_{1}} X_{i 2}^{B_{2}}+e_{i}$ & 283,7227 & 0,1957 & 0,3461 \\
\hline
\end{tabular}

